\title{
Spectral decompositions, ergodic averages, and the Hilbert transform
}

\author{
by \\ Earl Berkson (Urbana, IL) and T. A. Gillespie (Edinburgh)
}

\begin{abstract}
Let $U$ be a trigonometrically well-bounded operator on a Banach space $\mathfrak{X}$, and denote by $\left\{\mathfrak{A}_{n}(U)\right\}_{n=1}^{\infty}$ the sequence of $(C, 2)$ weighted discrete ergodic averages of $U$, that is,

$$
\mathfrak{A}_{n}(U)=\frac{1}{n} \sum_{0<|k| \leq n}\left(1-\frac{|k|}{n+1}\right) U^{k} .
$$

We show that this sequence $\left\{\mathfrak{A}_{n}(U)\right\}_{n=1}^{\infty}$ of weighted ergodic averages converges in the strong operator topology to an idempotent operator whose range is $\{x \in \mathfrak{X}: U x=x\}$, and whose null space is the closure of $(I-U) \mathfrak{X}$. This result expands the scope of the traditional Ergodic Theorem, and thereby serves as a link between Banach space spectral theory and ergodic operator theory. We also develop a characterization of trigonometrically well-bounded operators by their ability to "transfer" the discrete Hilbert transform to the Banach space setting via $(C, 1)$ weighting of Hilbert averages, and these results together with those on weighted ergodic averages furnish an explicit expression for the spectral decomposition of a trigonometrically well-bounded operator $U$ on a Banach space in terms of strong limits of appropriate averages of the powers of $U$. We also treat the special circumstances where corresponding results can be obtained with the $(C, 1)$ and $(C, 2)$ weights removed.
\end{abstract}

1. Introduction. The central theme of this note is the interplay between spectral decomposability and the boundedness and convergence properties of suitably weighted ergodic and Hilbert averages of an operator. It is shown that the use of $(C, 2)$ summability methods to weight these averages affords an expansion of ergodic operator theory via spectral theory. The main results are stated in Theorems 1.1, 5.2, 5.3, and 6.3.

2000 Mathematics Subject Classification: Primary 47A35, 47B40; Secondary 40G05, 40J05, 42A50.

Key words and phrases: trigonometrically well-bounded operator, spectral decomposition, ergodic averages, Cesàro means, Hilbert transform.

The work of the first author was partially supported by a grant from the National Science Foundation (U.S.A.). Both authors wish to thank the NATO Collaborative Research Grants Programme for its support. 
We begin by sketching the ingredients involved. Precise definitions will be given in the next section. Denote by $\mathfrak{B}(\mathfrak{X})$ the Banach algebra of all bounded linear operators from a Banach space $\mathfrak{X}$ into $\mathfrak{X}$, and let $I$ be the identity operator on $\mathfrak{X}$. An operator $U \in \mathfrak{B}(\mathfrak{X})$ is said to be trigonometrically well-bounded provided that $U$ can be represented in the form

$$
U=\int_{0-}^{2 \pi} e^{i t} d E(t)
$$

Here $E(\cdot)$ is an idempotent-valued function $\mathbb{R} \rightarrow \mathfrak{B}(\mathfrak{X})$ with certain additional properties weaker than those arising from a spectral measure (in particular, the strong left-hand limit $E\left(\lambda^{-}\right)$exists for all $\lambda \in \mathbb{R}$ ), and the integral exists as a Riemann-Stieltjes integral in the strong operator topology. These additional properties can be specified in spectral-theoretic terms so that $E(\cdot)$ is uniquely determined by $U$, and $E(\cdot)$ is then called the spectral decomposition of $U$.

We mention here that trigonometrically well-bounded operators occur frequently, since every invertible operator $U$ on a UMD space such that $U$ is power-bounded (that is, such that $\sup \left\{\left\|U^{k}\right\|: k \in \mathbb{Z}\right\}<\infty$ ) is automatically trigonometrically well-bounded (see [6], Theorem (4.5), or [4], Theorem (3.8).(i)). The UMD spaces are those Banach spaces with the unconditionality property for martingale differences (see inter alia [6] for further details) and are necessarily reflexive. On the other hand, there are many examples of trigonometrically well-bounded operators on reflexive Lebesgue spaces (in particular, on Hilbert space) which are not power-bounded (see, e.g., [5], Scholium (4.3) et seq.). Moreover, there is a trigonometrically wellbounded operator $U_{0}$ on a reflexive Banach space such that the sequence $\left\{(2 n)^{-1} \sum_{0<|k| \leq n} U_{0}^{k}\right\}_{n=1}^{\infty}$ of discrete ergodic averages for $U_{0}$ is not uniformly bounded (see Proposition 2.3 and item (3.13) below).

Our central result for ergodic averages (which will be established in Section 4) can be formulated as follows.

Theorem 1.1. Suppose that $\mathfrak{X}$ is a Banach space, and $U \in \mathfrak{B}(\mathfrak{X})$ is a trigonometrically well-bounded operator. Then

$$
\sup _{n \in \mathbb{N}, z \in \mathbb{T}}\left\|\frac{1}{n} \sum_{0<|k| \leq n}\left(1-\frac{|k|}{n+1}\right) z^{k} U^{k}\right\|<\infty,
$$

and the sequence

$$
\left\{\frac{1}{n} \sum_{0<|k| \leq n}\left(1-\frac{|k|}{n+1}\right) U^{k}\right\}_{n=1}^{\infty}
$$

converges in the strong operator topology of $\mathfrak{B}(\mathfrak{X})$ to an idempotent operator whose range is $\{x \in \mathfrak{X}: U x=x\}$, and whose null space is the closure of $(I-U) \mathfrak{X}$. 
Theorem 1.1 can be viewed as a spectral-theoretic relaxation of the power-boundedness condition used in the following version of the Mean Ergodic Theorem on reflexive spaces (see [10], Corollaries VIII.5.2 and VIII.5.4 for this formulation of the Mean Ergodic Theorem).

TheOrem 1.2. Let $\mathfrak{X}$ be a reflexive Banach space, and suppose that $U \in$ $\mathfrak{B}(\mathfrak{X})$ is an invertible operator which is power-bounded:

$$
\sup \left\{\left\|U^{k}\right\|: k=0, \pm 1, \pm 2, \ldots\right\}<\infty .
$$

Then the sequence

$$
\left\{\frac{1}{2 n} \sum_{0<|k| \leq n} U^{k}\right\}_{n=1}^{\infty}
$$

converges in the strong operator topology to the projection onto $\{x \in \mathfrak{X}$ : $U x=x\}$ along the closure of $(I-U) \mathfrak{X}$.

REMARK 1.1. Notice that the $n$th term of the sequence appearing in (1.3) is one-half of the $n$th $(C, 2)$ mean for the sequence $\left\{U^{j}+U^{-j}\right\}_{j=1}^{\infty}$. As in the scalar case, $(C, 1)$ summability implies $(C, 2)$ summability to the same value for sequences in a Banach space (adapt the discussion for the scalar case in [13], Theorem III.(1.21), p. 77, or [11], Theorem 2.11E, pp. 60, 61 ). On the other hand, if $U$ is a power-bounded invertible operator on a Banach space, then the convergence in the strong operator topology of the sequence $\left\{n^{-1} \sum_{0<|k| \leq n}(1-|k| /(n+1)) U^{k}\right\}_{n=1}^{\infty}$ implies the strong convergence of the sequence $\left\{(2 n)^{-1} \sum_{0<|k| \leq n} U^{k}\right\}_{n=1}^{\infty}$. This follows by standard arguments from Eberlein's theorem (see [12], p. 76 and the remark on p. 83 concerning its application). It is clear from these observations that Theorem 1.1 expands the scope of Theorem 1.2 in the direction of spectral theory. In this regard it is significant that, as mentioned above, the $(C, 1)$ averages $\left\{(2 n)^{-1} \sum_{0<|k| \leq n} U^{k}\right\}_{n=1}^{\infty}$ associated with a trigonometrically well-bounded operator $U$ on a reflexive space need not even be uniformly bounded in norm, let alone convergent in the strong operator topology.

After taking up the necessary estimates from harmonic analysis in Section 3, we finish the proof of Theorem 1.1 in Section 4, where we also take up some related results. In Section 5 we develop a characterization of trigonometrically well-bounded operators by their ability to "transfer" the discrete Hilbert transform to the Banach space setting via $(C, 1)$ weighting of the Hilbert averages (Theorem 5.2). This circle of ideas in conjunction with Corollary 4.4 leads to an explicit expression for the spectral decomposition of a trigonometrically well-bounded operator $U$ on a Banach space $\mathfrak{X}$ in terms of strong limits of appropriate averages for the powers of $U$ (Theorem 5.3). In Section 6, we treat the special circumstances which permit us 
to dispense with $(C, 2)$ weights for the ergodic averages and $(C, 1)$ weights for the Hilbert averages.

Throughout what follows, we shall freely use without explicit mention the elementary fact that for $\mathfrak{X}$ a Banach space and $\mathcal{S} \subseteq \mathfrak{B}(\mathfrak{X})$, the closure of $\mathcal{S}$ in the strong (respectively, weak) operator topology is compact in the strong (respectively, weak) operator topology if and only if for each $x \in \mathfrak{X}$ the closure of $\mathcal{S} x$ in the norm topology of $\mathfrak{X}$ (respectively, the weak topology of $\mathfrak{X}$ ) is compact in the norm topology of $\mathfrak{X}$ (respectively, the weak topology of $\mathfrak{X})$.

2. Background items from spectral theory. In this section, we recall the requisite features of trigonometrically well-bounded operators by starting out with the basic machinery of spectral families and their associated spectral integration.

Definition 2.1. A spectral family in a Banach space $\mathfrak{X}$ is an idempotent-valued function $E(\cdot): \mathbb{R} \rightarrow \mathfrak{B}(X)$ with the following properties:

(i) $E(\lambda) E(\tau)=E(\tau) E(\lambda)=E(\lambda)$ if $\lambda \leq \tau$;

(ii) $\sup \{\|E(\lambda)\|: \lambda \in \mathbb{R}\}<\infty$;

(iii) with respect to the strong operator topology, $E(\cdot)$ is right continuous and has a left-hand limit $E\left(\lambda^{-}\right)$at each point $\lambda \in \mathbb{R}$;

(iv) $E(\lambda) \rightarrow I$ as $\lambda \rightarrow \infty$ and $E(\lambda) \rightarrow 0$ as $\lambda \rightarrow-\infty$, each limit being with respect to the strong operator topology.

If, in addition, there exist $a, b \in \mathbb{R}$ with $a \leq b$ such that $E(\lambda)=0$ for $\lambda<a$ and $E(\lambda)=I$ for $\lambda \geq b, E(\cdot)$ is said to be concentrated on $[a, b]$.

Given a spectral family $E(\cdot)$ in $\mathfrak{X}$ concentrated on a compact interval $J=[a, b]$, an associated theory of spectral integration can be developed as follows. For each bounded function $\varphi: J \rightarrow \mathbb{C}$ and each partition $\mathcal{P}=$ $\left(\lambda_{0}, \lambda_{1}, \ldots, \lambda_{n}\right)$ of $J$, where we take $\lambda_{0}=a$ and $\lambda_{n}=b$, set

$$
\mathcal{S}(\mathcal{P} ; \varphi, E)=\sum_{k=1}^{n} \varphi\left(\lambda_{k}\right)\left\{E\left(\lambda_{k}\right)-E\left(\lambda_{k-1}\right)\right\} .
$$

If the net $\{\mathcal{S}(\mathcal{P} ; \varphi, E)\}$ converges in the strong operator topology of $\mathfrak{B}(\mathfrak{X})$ as $\mathcal{P}$ increases by refinement through the set of partitions of $J$, then the strong limit is called the spectral integral of $\varphi$ with respect to $E(\cdot)$ and is denoted by $\int_{J} \varphi(\lambda) d E(\lambda)$. In this case, we define $\int_{J}^{\oplus} \varphi(\lambda) d E(\lambda)$ by writing

$$
\int_{J}^{\oplus} \varphi(\lambda) d E(\lambda) \equiv \varphi(a) E(a)+\int_{J} \varphi(\lambda) d E(\lambda) .
$$

Denote by $\operatorname{BV}(J)$ the Banach algebra of functions $\varphi: J \rightarrow \mathbb{C}$ of bounded 
variation on $J$, with norm

$$
\|\varphi\|_{J}=|\varphi(b)|+\operatorname{var}_{J} \varphi .
$$

It can be shown that the spectral integral $\int_{J} \varphi(\lambda) d E(\lambda)$ exists for each $\varphi \in$ $\mathrm{BV}(J)$ and that the mapping $\varphi \mapsto \int_{J}^{\oplus} \varphi(\lambda) d E(\lambda)$ is an identity-preserving algebra homomorphism of $\mathrm{BV}(J)$ into $\mathfrak{B}(\mathfrak{X})$ satisfying

$$
\left\|\int_{J}^{\oplus} \varphi(t) d E(t)\right\| \leq\|\varphi\|_{J} \sup \{\|E(\lambda)\|: \lambda \in \mathbb{R}\} .
$$

(See [9], Chapter 17 or the simplified account in [3], §2.) In connection with these matters, we shall also consider the Banach algebra $B V(\mathbb{T})$, which consists of all $\psi: \mathbb{T} \rightarrow \mathbb{C}$ such that the function $\widetilde{\psi}(t) \equiv \psi\left(e^{i t}\right)$ belongs to $\mathrm{BV}([0,2 \pi])$, and which is furnished with the norm $\|\psi\|_{\mathrm{BV}(\mathbb{T})}=\|\widetilde{\psi}\|_{[0,2 \pi]}$.

Definition. An operator $U \in \mathfrak{B}(\mathfrak{X})$ is said to be trigonometrically well-bounded if there is a spectral family $E(\cdot)$ in $\mathfrak{X}$ concentrated on $[0,2 \pi]$ such that $U=\int_{[0,2 \pi]}^{\oplus} e^{i \lambda} d E(\lambda)$. In this case, it is possible to arrange that $E\left(2 \pi^{-}\right)=I$, and with this additional property the spectral family $E(\cdot)$ is uniquely determined by $U$, and is called the spectral decomposition of $U$.

This class of operators was introduced in [1], and its fundamental structural theory further developed in [2]. A characterization of trigonometrically well-bounded operators which is crucial for our considerations is stated as follows (see [1], Corollary 2.17, and [4], Corollary (2.3)). For a trigonometric polynomial $q$ given by $q(z)=\sum_{k=-N}^{N} \widehat{q}(k) z^{k}$ for all $z \in \mathbb{T}$, and an invertible operator $U \in \mathfrak{B}(\mathfrak{X})$, we shall henceforth symbolize by $q(U)$ the operator $\sum_{k=-N}^{N} \widehat{q}(k) U^{k}$. Note that, for a trigonometrically well-bounded operator $U$ with spectral decomposition $E(\cdot), U^{k}=\int_{[0,2 \pi]}^{\oplus} e^{i k \lambda} d E(\lambda)$ for each integer $k$ and hence $q(U)=\int_{[0,2 \pi]}^{\oplus} q\left(e^{i \lambda}\right) d E(\lambda)$ for each trigonometric polynomial $q$.

Theorem 2.1. Suppose that $\mathfrak{X}$ is a Banach space, and $U \in \mathfrak{B}(\mathfrak{X})$. Then $U$ is trigonometrically well-bounded if and only if $U$ is an invertible operator such that

the closure in the weak operator topology of $\{q(U): q$ is a trigonometric polynomial with $\left.\|q\|_{\mathrm{BV}(\mathbb{T})} \leq 1\right\}$ is compact in the weak operator topology.

If this is the case, then by the Uniform Boundedness Principle,

$$
\begin{array}{r}
\varrho(U) \equiv \sup \{\|q(U)\|: q \text { is a trigonometric polynomial } \\
\text { with } \left.\|q\|_{\mathrm{BV}(\mathbb{T})} \leq 1\right\}<\infty,
\end{array}
$$


and the spectral decomposition $E(\cdot)$ of $U$ satisfies

$$
\sup \{\|E(\lambda)\|: \lambda \in \mathbb{R}\} \leq 3 \varrho(U) .
$$

When $\mathfrak{X}$ is reflexive, and $U$ is invertible, the boundedness condition in (2.3) is necessary and sufficient for $U$ to be trigonometrically well-bounded.

Corollary 2.2. If $U \in \mathfrak{B}(\mathfrak{X})$ is trigonometrically well-bounded, then $z U$ is trigonometrically well-bounded for every $z \in \mathbb{T}$.

Proof. For each $z \in \mathbb{T}$,

$\left\{q(z U): q\right.$ is a trigonometric polynomial with $\left.\|q\|_{\mathrm{BV}(\mathbb{T})} \leq 1\right\}$

$\subseteq 2\left\{q(U): q\right.$ is a trigonometric polynomial with $\left.\|q\|_{\mathrm{BV}(\mathbb{T})} \leq 1\right\}$.

To round out the picture presented by Theorem 2.1, we mention the following consequence of the reasoning involved in [2], Example (3.1).

Proposition 2.3. There is a trigonometrically well-bounded operator $U_{0}$ on a reflexive Banach space $\mathfrak{X}_{0}$ such that for every trigonometric polynomial $q$,

$$
\left\|q\left(U_{0}\right)\right\| \geq\|q\|_{\mathrm{BV}(\mathbb{T})} .
$$

Combining either of (2.1) or (2.3) with Proposition 2.3, we arrive at the following characterization for boundedness in $\mathrm{BV}(\mathbb{T})$ of sequences of trigonometric polynomials.

Proposition 2.4. Suppose that $\left\{q_{n}\right\}_{n=1}^{\infty}$ is a sequence of trigonometric polynomials. The following two assertions are equivalent:

(i) $\left\{q_{n}\right\}_{n=1}^{\infty}$ is bounded in $\mathrm{BV}(\mathbb{T})$.

(ii) Whenever $U$ is a trigonometrically well-bounded operator on a $B a$ nach space $\mathfrak{X}$, the sequence $\left\{q_{n}(U)\right\}_{n=1}^{\infty}$ is uniformly bounded in $\mathfrak{B}(\mathfrak{X})$.

Our next item deals with the counterpart of (2.2) in the strong operator topology. Specifically, the following proposition holds (compare [9], Theorem 17.14).

Proposition 2.5. The statement of Theorem 2.1 remains valid if, throughout (2.2), we replace the weak operator topology by the strong operator topology.

Since the weak operator topology is contained in the strong operator topology, it is clear that (2.2) follows from the corresponding assertion in the strong operator topology. In order to establish Proposition 2.5, by showing conversely that this corresponding assertion for the strong operator topology holds if $U$ is trigonometrically well-bounded, we first need to consider the following pleasant property of spectral families of projections, as well as some convexity notions which will be advantageous from here on. 
Proposition 2.6. Let $\mathcal{E}(\cdot)$ be a spectral family of projections in a Banach space $\mathfrak{X}$. Then for each $x \in \mathfrak{X},\{\mathcal{E}(t) x: t \in \mathbb{R}\}$ is totally bounded.

Proof (see [9], p. 347, for an alternative argument). It is elementary that any sequence $\left\{t_{n}\right\}_{n=1}^{\infty} \subseteq \mathbb{R}$ has a monotone subsequence $\left\{t_{n_{k}}\right\}_{k=1}^{\infty}$. The convergence of $\left\{\mathcal{E}\left(t_{n_{k}}\right) x\right\}_{k=1}^{\infty}$ in the norm topology follows from properties (iii) and (iv) in Definition 2.1 for a spectral family of projections.

Definition 2.2. For a subset $\mathcal{S}$ of a Banach space $\mathfrak{X}$, we denote by $\mathbb{T} \mathcal{S}$ the set $\{z s: z \in \mathbb{T}, s \in \mathcal{S}\}$, and we define $\operatorname{aco}(\mathcal{S})$, the absolutely convex hull of $\mathcal{S}$, by writing

$$
\begin{aligned}
\operatorname{aco}(\mathcal{S}) & =\left\{\sum_{j=1}^{N} \alpha_{j} x_{j}: N \in \mathbb{N},\left\{\alpha_{j}\right\}_{j=1}^{N} \subseteq \mathbb{C},\left\{x_{j}\right\}_{j=1}^{N} \subseteq \mathcal{S}, \sum_{j=1}^{N}\left|\alpha_{j}\right| \leq 1\right\} \\
& =\left\{\sum_{j=1}^{N} \alpha_{j} x_{j}: N \in \mathbb{N},\left\{\alpha_{j}\right\}_{j=1}^{N} \subseteq \mathbb{C},\left\{x_{j}\right\}_{j=1}^{N} \subseteq \mathcal{S}, \sum_{j=1}^{N}\left|\alpha_{j}\right|=1\right\} \\
& =\operatorname{co}(\mathbb{T} \mathcal{S}),
\end{aligned}
$$

where $\operatorname{co}(\cdot)$ denotes the usual convex hull.

Notice that if the set $\mathcal{S}$ appearing in (2.5) has compact closure in the norm topology of $\mathfrak{X}$ (respectively, in the weak topology of $\mathfrak{X}$ ), then it is clear from the right-hand side of (2.5) together with Mazur's Theorem in [10], Theorem V.2.6 (respectively, together with the Krein-Šmulian Theorem in [10], Theorem V.6.4) that, with respect to the norm topology of $\mathfrak{X}$ (respectively, the weak topology of $\mathfrak{X}), \operatorname{aco}(\mathcal{S})$ has compact closure. Such facts will be used in what follows without explicit mention.

Proof of Proposition 2.5. Suppose that $U \in \mathfrak{B}(\mathfrak{X})$ is trigonometrically well-bounded, and let $E(\cdot)$ be the spectral decomposition of $U$. If $q$ is a trigonometric polynomial such that $\|q\|_{\mathrm{BV}(\mathbb{T})} \leq 1$, and $x \in \mathfrak{X}$, then, letting $u=\left(t_{0}, t_{1}, \ldots, t_{n}\right)$ increase by refinement through the partitions of the interval $[0,2 \pi]$, we see with the aid of a summation by parts that

$$
\begin{aligned}
q(U) x & =\int_{[0,2 \pi]}^{\oplus} q\left(e^{i t}\right) d E(t) x \\
& =\lim _{u}\left\{q(1) E(0) x+\sum_{j=1}^{n} q\left(e^{i t_{j}}\right)\left[E\left(t_{j}\right)-E\left(t_{j-1}\right)\right] x\right\} \\
& =\lim _{u}\left\{q(1) x-\sum_{j=1}^{n}\left[q\left(e^{i t_{j}}\right)-q\left(e^{i t_{j-1}}\right)\right] E\left(t_{j-1}\right) x\right\} .
\end{aligned}
$$

Observing the form of the expression in brackets of the right-hand member, and taking into account the assumption that $\|q\|_{\mathrm{BV}(\mathbb{T})} \leq 1$, we infer that, 
with respect to the norm topology of $\mathfrak{X}$,

$$
q(U) x \in \text { the closure of aco }\{E(t) x: t \in \mathbb{R}\} .
$$

The proof of Proposition 2.5 can now be readily completed by making use of Proposition 2.6.

In closing this section we take up a result which will play a pivotal role in the characterization of trigonometrically well-bounded operators by their interplay with the discrete Hilbert transform (Theorem 5.2 below).

Lemma 2.7. Let $\mathfrak{X}$ be a Banach space, and suppose that $T \in \mathfrak{B}(\mathfrak{X})$ is invertible. Let

$$
\mathcal{W}=\left\{\sum_{0<|k| \leq n}\left(1-\frac{|k|}{n+1}\right) \frac{z^{k} T^{k}}{k}: n \in \mathbb{N}, z \in \mathbb{T}\right\},
$$

and let

$$
\mathcal{D}=\{z \in \mathbb{C}:|z| \leq 1\} .
$$

Then for each trigonometric polynomial $q$ such that $\|q\|_{\mathrm{BV}(\mathbb{T})} \leq 1$,

$$
q(T) \in \mathcal{D} I+\operatorname{ucl}\{\operatorname{aco}(\mathcal{W})\},
$$

where ucl denotes closure in the uniform operator topology. Moreover, if

then

$$
\mathfrak{s} \equiv \sup \{\|S\|: S \in \mathcal{W}\}<\infty
$$

$$
\begin{array}{r}
\sup \left\{\|q(T)\|: q \text { is a trigonometric polynomial with }\|q\|_{\mathrm{BV}(\mathbb{T})} \leq 1\right\} \\
\leq 1+\frac{\mathfrak{s}}{2 \pi} .
\end{array}
$$

Proof. Our method of proof will be an adaptation of the reasoning used on pp. 773-774 of [2]. Choose $N \in \mathbb{N}$ such that $\widehat{q}(k)=0$ for $|k|>N$. We see with the aid of an integration by parts that for $n \geq N$,

$$
\begin{aligned}
q(T) & =\widehat{q}(0) I+\frac{1}{2 \pi} \int_{0}^{2 \pi} q\left(e^{i t}\right) \sum_{0<|k| \leq n} e^{-i k t} T^{k} d t \\
& =\widehat{q}(0) I-\frac{i}{2 \pi} \int_{0}^{2 \pi} \sum_{0<|k| \leq n} \frac{e^{-i k t}}{k} T^{k} d q\left(e^{i t}\right) .
\end{aligned}
$$

Since $\widehat{q}(k)=0$ for $|k|>N$, we find after simple manipulations that the integral on the right of (2.8) equals

$$
\begin{aligned}
\int_{0}^{2 \pi} \sum_{0<|k| \leq n}\left(1-\frac{|k|}{n+1}\right) & \frac{e^{-i k t}}{k} T^{k} d q\left(e^{i t}\right) \\
& +\frac{1}{n+1} \int_{0}^{2 \pi} \sum_{0<|k| \leq N} \operatorname{sgn}(k) e^{-i k t} T^{k} d q\left(e^{i t}\right),
\end{aligned}
$$


where sgn denotes the signum. Substituting (2.9) on the right of (2.8), we obtain

$$
\begin{aligned}
q(T)= & \widehat{q}(0) I+\int_{0}^{2 \pi} \frac{1}{2 \pi i} \sum_{0<|k| \leq n}\left(1-\frac{|k|}{n+1}\right) \frac{e^{-i k t}}{k} T^{k} d q\left(e^{i t}\right) \\
& -\frac{i}{2 \pi(n+1)} \int_{0}^{2 \pi} \sum_{0<|k| \leq N} \operatorname{sgn}(k) e^{-i k t} T^{k} d q\left(e^{i t}\right) .
\end{aligned}
$$

Since $\|q\|_{\mathrm{BV}(\mathbb{T})} \leq 1$, the first integral on the right of (2.10) clearly belongs to $\operatorname{ucl}\{\operatorname{aco}(\mathcal{W})\}$. As $n \rightarrow \infty$, the last term on the right of $(2.10)$ obviously tends to 0 in the uniform operator topology, because $N$ is fixed. The conclusion in (2.6) now follows by letting $n \rightarrow \infty$ on the right of (2.10), and noting that $|\widehat{q}(0)| \leq 1$.

The conclusion in (2.7) can be seen by observing that the first integral on the right of (2.10) has operator norm not exceeding $\mathfrak{s} /(2 \pi)$.

Corollary 2.8. Let $\mathfrak{X}$ be a Banach space, and suppose that $T \in \mathfrak{B}(\mathfrak{X})$ is invertible. Let $\mathcal{W}$ and $\mathcal{D}$ be as defined in the statement of Lemma 2.7. Then for each $x \in \mathfrak{X}$, and each trigonometric polynomial $q$ such that $\|q\|_{\mathrm{BV}(\mathbb{T})}$ $\leq 1$, we have

$$
q(T) x \in \mathcal{D} x+\mathfrak{X}^{*}-\operatorname{cl}\{\operatorname{aco}(\mathcal{W} x)\},
$$

where the symbol $\mathfrak{X}^{*}$-cl denotes closure in the weak topology of $\mathfrak{X}$.

3. Variational estimates with trigonometric polynomials. In all that follows, let $U$ be a trigonometrically well-bounded operator on a Banach space $\mathfrak{X}$, and let $E(\cdot)$ be the spectral decomposition of $U$. In this section we shall be concerned with estimating, via $\|q\|_{\mathrm{BV}(\mathbb{T})}$, expressions of the form $\|q(U)\|$, where $q$ runs through a variety of special trigonometric polynomials closely associated with averaging methods for the powers of $U$, that is, with summability methods needed for our main results. In view of (2.1), Theorem 2.1, and Proposition 2.4, a principal outcome of our considerations in this section can be summarized by the assertion that in order to have universal theorems about the strong convergence of discrete ergodic averages associated with trigonometrically well-bounded operators, it is necessary to deal with weighted ergodic averages such as the averages exhibited in (1.3). The next section will deal with the converse circle of ideas, including the proof of Theorem 1.1.

We shall denote by $\left\{D_{n}\right\}_{n=0}^{\infty}$ and $\left\{\kappa_{n}\right\}_{n=0}^{\infty}$, respectively, the Dirichlet kernel and the Fejér kernel for $\mathbb{T}$, which are defined for all integers $n \geq 0$ and all $z=e^{i t}(t \in \mathbb{R})$ by 


$$
\begin{aligned}
D_{n}(z) & =\sum_{k=-n}^{n} z^{k}=\frac{\sin ((n+1 / 2) t)}{\sin (t / 2)} \\
\kappa_{n}(z) & =\frac{1}{n+1} \sum_{j=0}^{n} D_{j}(z) \\
& =\sum_{k=-n}^{n}\left(1-\frac{|k|}{n+1}\right) z^{k}=\frac{1}{n+1}\left\{\frac{\sin ((n+1) t / 2)}{\sin (t / 2)}\right\}^{2} .
\end{aligned}
$$

The right-hand members of (3.1) and (3.2) are to be interpreted by continuity when $t \in(2 \pi) \mathbb{Z}: D_{n}(1)=2 n+1$, and

$$
\kappa_{n}(1)=n+1
$$

As is well known, $\left\{\kappa_{n}\right\}_{n=0}^{\infty}$ is an approximate identity for $L^{1}(\mathbb{T})$ such that $\kappa_{n} \geq 0,(2 \pi)^{-1} \int_{0}^{2 \pi} \kappa_{n}\left(e^{i t}\right) d t=1$, and

$$
\kappa_{n}\left(e^{i t}\right) \leq \min \left\{n+1, \frac{\pi^{2}}{(n+1) t^{2}}\right\} \quad \text { for } 0<|t| \leq \pi,
$$

whereas (see, for instance, [13], II.(12.1))

$$
\lim _{n \rightarrow \infty} \frac{(2 \pi)^{-1} \int_{0}^{2 \pi}\left|D_{n}\left(e^{i t}\right)\right| d t}{\log n}=\frac{4}{\pi^{2}} .
$$

Let $h: \mathbb{Z} \rightarrow \mathbb{R}$ be the discrete Hilbert kernel specified by $h(k)=1 / k$ for $k \in \mathbb{Z} \backslash\{0\}$ and $h(0)=0$. Thus, the discrete Hilbert transform is classically defined by convolution with $h$. The spectral theory of trigonometrically wellbounded operators is closely linked to the discrete Hilbert transform (see [2], Theorem (2.4), and its extensions to the general Banach space setting developed in Section 5 below). For our purposes this linkage can ultimately be attributed to the fact that $h=\widehat{\phi}$, where $\phi \in \mathrm{BV}(\mathbb{T})$ is defined by

$$
\phi\left(e^{i t}\right)=i(\pi-t) \quad \text { for } 0<t<2 \pi, \quad \text { and } \quad \phi(1)=0 .
$$

We now describe some trigonometric polynomials associated with this circle of ideas. For $n \in \mathbb{N}$, we shall henceforth denote by $s_{n}$ and $\sigma_{n}$, respectively, the $n$th partial sum and the $n$th Cesàro mean of the Fourier series for $\phi$, and so for all $z \in \mathbb{T}$,

$$
\begin{aligned}
s_{n}(z) & =\sum_{0<|k| \leq n} \frac{z^{k}}{k} \\
\sigma_{n}(z) & =\left(\kappa_{n} * \phi\right)(z)=\sum_{0<|k| \leq n}\left(1-\frac{|k|}{n+1}\right) \frac{z^{k}}{k} \\
& =s_{n}(z)-\frac{1}{n+1} \sum_{0<|k| \leq n} \operatorname{sgn}(k) z^{k}
\end{aligned}
$$


where $\operatorname{sgn}(k)$ denotes the signum of $k$. Notice with the aid of (3.5) that there is a positive absolute constant $\eta$ such that for all $n \in \mathbb{N}$,

$$
\operatorname{var}\left(s_{n}, \mathbb{T}\right)=\int_{0}^{2 \pi}\left|\frac{d s_{n}\left(e^{i t}\right)}{d t}\right| d t \geq 2 \pi\left(\left\|D_{n}\right\|_{L^{1}(\mathbb{T})}-1\right) \geq 2 \pi(\eta \log n-1),
$$

and so $\operatorname{var}\left(s_{n}, \mathbb{T}\right) \rightarrow \infty$ as $n \rightarrow \infty$. By contrast,

$$
\operatorname{var}\left(\sigma_{n}, \mathbb{T}\right)=\operatorname{var}\left(\kappa_{n} * \phi, \mathbb{T}\right) \leq \operatorname{var}(\phi, \mathbb{T})=4 \pi,
$$

and hence

$$
\sup _{n \in \mathbb{N}}\left\|\sigma_{n}\right\|_{\mathrm{BV}(\mathbb{T})} \leq 4 \pi .
$$

In the light of Proposition 2.4, the disparate behavior in BV(T) evinced by (3.9) and (3.10) renders $\left\{\sigma_{n}\right\}_{n=1}^{\infty}$ more suitable than $\left\{s_{n}\right\}_{n=1}^{\infty}$ for treating the general theory of trigonometrically well-bounded operators.

For later use in conjunction with the weighted discrete ergodic averages occurring in (1.3) we next consider two further sequences of trigonometric polynomials, $\left\{\tau_{n}\right\}_{n=1}^{\infty}$ and $\left\{\mathfrak{A}_{n}\right\}_{n=1}^{\infty}$, defined by writing, for each $n \in \mathbb{N}$ and $z \in \mathbb{T}$,

$$
\tau_{n}(z)=\frac{1}{n} \sum_{0<|k| \leq n} z^{k}=\frac{1}{n}\left(D_{n}(z)-1\right),
$$

$$
\mathfrak{A}_{n}(z)=\frac{1}{n} \sum_{0<|k| \leq n}\left(1-\frac{|k|}{n+1}\right) z^{k}=\left(\kappa_{n} * \tau_{n}\right)(z)=\frac{1}{n}\left(\kappa_{n}(z)-1\right) .
$$

For each $n \in \mathbb{N}, \operatorname{var}\left(\tau_{n}, \mathbb{T}\right)=n^{-1} \operatorname{var}\left(D_{n}, \mathbb{T}\right)$. Temporarily fix $n \in \mathbb{N}$, and, for $j=1, \ldots, n$, let

$$
t_{j}=\frac{j \pi}{n+1 / 2}, \quad u_{j}=\frac{\pi / 2+j \pi}{n+1 / 2} .
$$

Thus, $0<t_{j}<u_{j} \leq \pi, u_{j}<t_{j+1}$, and $\sin \left((n+1 / 2) t_{j}\right)=0$, while $\left|\sin \left((n+1 / 2) u_{j}\right)\right|=1$. It is now easy to see that

$$
\begin{aligned}
\operatorname{var}\left(D_{n}, \mathbb{T}\right) & \geq \sum_{j=1}^{n}\left|D_{n}\left(e^{i t_{j}}\right)-D_{n}\left(e^{i u_{j}}\right)\right|=\sum_{j=1}^{n}\left|D_{n}\left(e^{i u_{j}}\right)\right| \\
& \geq \frac{2 n+1}{\pi} \sum_{j=2}^{n+1} \frac{1}{j} \geq \frac{2 n+1}{\pi} \log \left(\frac{n+2}{2}\right),
\end{aligned}
$$

and consequently $\operatorname{var}\left(\tau_{n}, \mathbb{T}\right) \rightarrow \infty$ as $n \rightarrow \infty$, because

$$
\operatorname{var}\left(\tau_{n}, \mathbb{T}\right)=n^{-1} \operatorname{var}\left(D_{n}, \mathbb{T}\right)>\frac{2}{\pi} \log \left(\frac{n+2}{2}\right) .
$$

On the other hand, it is evident from the right-hand member of (3.11) that for each $n \in \mathbb{N}, \operatorname{var}\left(\mathfrak{A}_{n}, \mathbb{T}\right)=n^{-1} \operatorname{var}\left(\kappa_{n}, \mathbb{T}\right)$. By Bernstein's Inequality 
formulated for estimating in the norm of $L^{p}(\mathbb{T}), 1 \leq p \leq \infty$ (easily deducible from, e.g., the method of proof for Proposition $\mathrm{B}^{\prime}$ in [7]), we find by taking $p=1$ that

$$
\frac{1}{2 \pi} \int_{0}^{2 \pi}\left|\frac{d \kappa_{n}\left(e^{i t}\right)}{d t}\right| d t \leq \frac{n}{2 \pi} \int_{0}^{2 \pi} \kappa_{n}\left(e^{i t}\right) d t=n
$$

So $\operatorname{var}\left(\mathfrak{A}_{n}, \mathbb{T}\right) \leq 2 \pi$. Since $\left|\mathfrak{A}_{n}(1)\right|=1$, we now have, in contrast to (3.13),

$$
\sup _{n \in \mathbb{N}}\left\|\mathfrak{A}_{n}\right\|_{\mathrm{BV}(\mathbb{T})} \leq 2 \pi+1 .
$$

Temporarily fix a point $w \in \mathbb{T}$, and for each $n \in \mathbb{N}$, define the trigonometric polynomial $\psi_{n}$ by putting $\psi_{n}(z)=\mathfrak{A}_{n}(w z)$ for all $z \in \mathbb{T}$. Thus, $\operatorname{var}\left(\psi_{n}, \mathbb{T}\right)=$ $\operatorname{var}\left(\mathfrak{A}_{n}, \mathbb{T}\right) \leq 2 \pi$, and, from (3.14), we have

$$
\left|\psi_{n}(1)\right|=\left|\mathfrak{A}_{n}(w)\right| \leq\left\|\mathfrak{A}_{n}\right\|_{\mathrm{BV}(\mathbb{T})} \leq 2 \pi+1 .
$$

Upon combining (3.15) and the estimate for $\operatorname{var}\left(\psi_{n}, \mathbb{T}\right)$ with $(2.1)$, we have now established the first conclusion of Theorem 1.1, which we state here with the following more specific estimate.

Theorem 3.1. Suppose that $\mathfrak{X}$ is a Banach space, and $U \in \mathfrak{B}(\mathfrak{X})$ is a trigonometrically well-bounded operator with decomposition of the identity $E(\cdot)$. Then

$$
\sup _{n \in \mathbb{N}, z \in \mathbb{T}}\left\|\frac{1}{n} \sum_{0<|k| \leq n}\left(1-\frac{|k|}{n+1}\right) z^{k} U^{k}\right\| \leq(4 \pi+1) \sup \{\|E(\lambda)\|: \lambda \in \mathbb{R}\} .
$$

We conclude this section with some further results on trigonometric polynomials allied to the averaging sequences for operators $\left\{\tau_{n}(U)\right\}_{n=1}^{\infty}$ and $\left\{\mathfrak{A}_{n}(U)\right\}_{n=1}^{\infty}$. Our focus now will be on one-sided averages. For this purpose, let us define, for each $n \in \mathbb{N}$ and all $z \in \mathbb{T}$,

$$
\begin{aligned}
& \mathfrak{t}_{n}(z)=\frac{1}{n} \sum_{k=1}^{n} z^{k}, \\
& \mathfrak{a}_{n}(z)=\frac{1}{n} \sum_{k=1}^{n}\left(1-\frac{k}{n+1}\right) z^{k} .
\end{aligned}
$$

Denote by the symbols $\Re(\cdot)$ and $\Im(\cdot)$, respectively, the real and imaginary parts of a complex number. Examination of the left and right members of (3.8) and application of (3.9) together with (3.10) show that

$$
\sup _{n \in \mathbb{N}}\left\|\Im\left(\mathfrak{t}_{n}\right)\right\|_{\mathrm{BV}(\mathbb{T})}=\infty .
$$

Since $\tau_{n}=2 \Re\left(\mathfrak{t}_{n}\right)$, we can restate (3.13) accordingly, and summarize as follows our observations concerning the behavior in $\mathrm{BV}(\mathbb{T})$ of the sequence $\left\{\mathfrak{t}_{n}\right\}_{n=1}^{\infty}$. 
Proposition 3.2. For the sequence $\left\{\mathfrak{t}_{n}\right\}_{n=1}^{\infty}$ of trigonometric polynomials defined in (3.16) we have

$$
\sup _{n \in \mathbb{N}}\left\|\Re\left(\mathfrak{t}_{n}\right)\right\|_{\mathrm{BV}(\mathbb{T})}=\sup _{n \in \mathbb{N}}\left\|\Im\left(\mathfrak{t}_{n}\right)\right\|_{\mathrm{BV}(\mathbb{T})}=\infty .
$$

The situation with the sequence $\left\{\mathfrak{a}_{n}\right\}_{n=1}^{\infty}$ of trigonometric polynomials in (3.17) is more felicitous. If we convolve the left and right members of (3.8) with $\kappa_{n}$ and transpose, we find that for each $n \in \mathbb{N}$ and all $z \in \mathbb{T}$,

$2 i \Im\left(\mathfrak{a}_{n}(z)\right)=\frac{1}{n} \sum_{0<|k| \leq n}\left(1-\frac{|k|}{n+1}\right) \operatorname{sgn}(k) z^{k}=\frac{n+1}{n}\left(\sigma_{n}(z)-\left(\kappa_{n} * \sigma_{n}\right)(z)\right)$.

From the equality on the right here, it follows with the aid of (3.10) that for each $n \in \mathbb{N}$,

$$
\begin{aligned}
\| \frac{1}{n} \sum_{0<|k| \leq n} & \left(1-\frac{|k|}{n+1}\right) \operatorname{sgn}(k) z^{k} \|_{\mathrm{BV}(\mathbb{T})} \\
& =\operatorname{var}\left(\frac{1}{n} \sum_{0<|k| \leq n}\left(1-\frac{|k|}{n+1}\right) \operatorname{sgn}(k) z^{k}, \mathbb{T}\right) \leq 16 \pi .
\end{aligned}
$$

Since $\mathfrak{A}_{n}=2 \Re\left(\mathfrak{a}_{n}\right)$, we can combine (3.18) with (3.14) to obtain

$$
\sup _{n \in \mathbb{N}}\left\|\mathfrak{a}_{n}\right\|_{\mathrm{BV}(\mathbb{T})} \leq 9 \pi+1 / 2 .
$$

4. Convergence of the weighted ergodic averages for trigonometrically well-bounded operators. In order to convert (3.14) to the second conclusion of Theorem 1.1 regarding strong convergence of averages, we shall rely on the following two additional background items from the theory of trigonometrically well-bounded operators (see [3], Proposition (2.10) and Theorem (3.14)).

TheOREM 4.1. Let $\mathfrak{X}$ be a Banach space, and suppose that $\mathcal{E}(\cdot)$ is a spectral family of projections in $\mathfrak{X}$ concentrated on a compact interval $[u, v]$. Let $\left\{g_{\alpha}\right\}$ be a net in $\mathrm{BV}([u, v])$, and let $g$ be a complex-valued function on $[u, v]$ such that

(i) $\sup _{\alpha} \operatorname{var}\left(g_{\alpha},[u, v]\right)<\infty$,

(ii) $g_{\alpha} \rightarrow g$ pointwise on $[u, v]$.

Then $g \in \mathrm{BV}([u, v])$, and the net $\left\{\int_{[u, v]}^{\oplus} g_{\alpha} d \mathcal{E}\right\}$ converges in the strong operator topology of $\mathfrak{B}(\mathfrak{X})$ to $\int_{[u, v]}^{\oplus} g d \mathcal{E}$.

THEOREM 4.2. Let $U$ be a trigonometrically well-bounded operator on a Banach space $\mathfrak{X}$, and let $E(\cdot)$ be the spectral decomposition of $U$. Then for $0 \leq \lambda<2 \pi$ we have: 
(i) $\left\{E(\lambda)-E\left(\lambda^{-}\right)\right\} \mathfrak{X}=\left\{x \in \mathfrak{X}: U x=e^{i \lambda} x\right\}$,

(ii) $\left[I-\left\{E(\lambda)-E\left(\lambda^{-}\right)\right\}\right] \mathfrak{X}$ is the closure of $\left(e^{i \lambda} I-U\right) \mathfrak{X}$.

The inequality in (1.2) was disposed of by Theorem 3.1; in view of Theorem 4.2 , the remaining conclusion of Theorem 1.1 is covered by the following result.

THEOREM 4.3. Let $U$ be a trigonometrically well-bounded operator on a Banach space $\mathfrak{X}$, and let $E(\cdot)$ be the spectral decomposition of $U$. Then, in the notation of (3.11) and (3.17), each of the sequences $\left\{\mathfrak{A}_{n}(U)\right\}_{n=1}^{\infty}$ and $\left\{2 \mathfrak{a}_{n}(U)\right\}_{n=1}^{\infty}$ converges to $E(0)$ in the strong operator topology of $\mathfrak{B}(\mathfrak{X})$.

Proof. Let $\mathcal{C}$ denote the characteristic function, relative to $\mathbb{T}$, of the singleton set $\{1\}$. It is clear from the right member of (3.2), (3.3), and the expression on the right of (3.11) that the sequence of trigonometric polynomials

$$
\left\{\mathfrak{A}_{n}\right\}_{n=1}^{\infty} \text { tends pointwise on } \mathbb{T} \text { to } \mathcal{C} .
$$

For $z \in \mathbb{T}$, it is elementary that

$$
\lim _{n} \frac{1}{n} \sum_{j=1}^{n} z^{j}=\mathcal{C}(z)
$$

and hence the sequence $\left\{2 \mathfrak{a}_{n}\right\}_{n=1}^{\infty}$ also tends pointwise on $\mathbb{T}$ to $\mathcal{C}$, since the $(C, 1)$ summability of the sequence $\left\{z_{j}\right\}_{j=1}^{\infty}$ implies its $(C, 2)$ summability to the same value.

From (4.1) we know that on the interval $0 \leq t \leq 2 \pi,\left\{\mathfrak{A}_{n}\left(e^{i t}\right)\right\}_{n=1}^{\infty}$ tends pointwise to $\chi$, the characteristic function, relative to $[0,2 \pi]$, of the doubleton set $\{0,2 \pi\}$. Taking (3.14) into account, we can invoke Theorem 4.1 to infer that, with convergence in the strong operator topology,

$$
\mathfrak{A}_{n}(U)=\int_{[0,2 \pi]}^{\oplus} \mathfrak{A}_{n}\left(e^{i t}\right) d E(t) \rightarrow \int_{[0,2 \pi]}^{\oplus} \chi(t) d E(t) .
$$

Since the definition of the spectral decomposition for a trigonometrically well-bounded operator requires that $E\left((2 \pi)^{-}\right)=I$, it is easy to see directly that $\int_{[0,2 \pi]}^{\oplus} \chi(t) d E(t)=E(0)$. Using this fact in (4.2) gives the desired conclusion for $\left\{\mathfrak{A}_{n}(U)\right\}_{n=1}^{\infty}$. A similar argument based on (3.19) establishes the remaining conclusion for $\left\{2 \mathfrak{a}_{n}(U)\right\}_{n=1}^{\infty}$.

COROLlary 4.4. Under the hypotheses of Theorem 4.3, for each $\lambda$ such that $0 \leq \lambda<2 \pi$, we have, with convergence in the strong operator topology of $\mathfrak{B}(\mathfrak{X})$,

$$
\frac{1}{n} \sum_{0<|k| \leq n}\left(1-\frac{|k|}{n+1}\right) e^{-i k \lambda} U^{k} \rightarrow E(\lambda)-E\left(\lambda^{-}\right),
$$




$$
\frac{2}{n} \sum_{k=1}^{n}\left(1-\frac{k}{n+1}\right) e^{-i k \lambda} U^{k} \rightarrow E(\lambda)-E\left(\lambda^{-}\right) .
$$

Proof. By Corollary 2.2, for each $z \in \mathbb{T}$, the operator $z U$ is also trigonometrically well-bounded. Denoting the spectral decomposition of $z U$ by $E_{z}(\cdot)$, and taking $z=e^{-i \lambda}$, we infer from Theorem 4.3 that, in the strong operator topology,

$$
\frac{1}{n} \sum_{0<|k| \leq n}\left(1-\frac{|k|}{n+1}\right) e^{-i k \lambda} U^{k} \rightarrow E_{z}(0) .
$$

However, Theorem 4.2 shows that the idempotent operators $E_{z}(0)$ and $E(\lambda)-E\left(\lambda^{-}\right)$have the same range and the same null space, and so coincide. It is now clear that (4.3) holds, and similar reasoning establishes (4.4).

The results of Theorem 4.3 and Corollary 4.4 specialize to the formalism of traditional mean ergodic theorems if the appropriate one-sided or twosided boundedness of the powers of $U$ is assumed. This follows by standard summability arguments involving Eberlein's theorem (cf. the observations concerning Eberlein's theorem in Remark 1.1), although, in the present context, simpler arguments are available (by invoking the one-sided result in [3], Corollary (3.17)). We state as follows the power-boundedness theorem corresponding to Corollary 4.4.

THEOREM 4.5. Let $U$ be a trigonometrically well-bounded operator on a Banach space $\mathfrak{X}$, let $E(\cdot)$ be the spectral decomposition of $U$, and suppose that $0 \leq \lambda<2 \pi$.

(i) If $\sup \left\{\left\|U^{k}\right\|: k \in \mathbb{Z}\right\}<\infty$, then

$$
\frac{1}{2 n} \sum_{0<|k| \leq n} e^{-i k \lambda} U^{k} \rightarrow E(\lambda)-E\left(\lambda^{-}\right)
$$

in the strong operator topology.

(ii) If $\sup \left\{\left\|U^{k}\right\|: k \in \mathbb{Z}, k \geq 1\right\}<\infty$, then

$$
\frac{1}{n} \sum_{k=1}^{n} e^{-i k \lambda} U^{k} \rightarrow E(\lambda)-E\left(\lambda^{-}\right)
$$

in the strong operator topology.

Taking $\lambda=0$ in Theorem 4.5, we obtain the strengthened conclusions of Theorem 4.3 under additional power-boundedness assumptions. Specifically, if $U$ is a trigonometrically well-bounded operator and $\sup \left\{\left\|U^{k}\right\|\right.$ : $k \in \mathbb{Z}\}<\infty\left(\right.$ resp. $\left.\sup \left\{\left\|U^{k}\right\|: k \in \mathbb{Z}, k \geq 1\right\}<\infty\right)$, then the ergodic means $\left\{(2 n)^{-1} \sum_{0<|k| \leq n} U^{k}\right\}_{n=1}^{\infty}$ (resp. $\left\{n^{-1} \sum_{k=1}^{n} U^{k}\right\}_{n=1}^{\infty}$ ) converge in the strong 
operator topology to $E(0)$. In particular, the conclusion of Theorem 1.2 is valid without the reflexivity assumption on $\mathfrak{X}$ provided the power-bounded operator $U$ is trigonometrically well-bounded.

5. The discrete Hilbert transform in spectral theory. In this section we take up the characterization of trigonometrically well-bounded operators by their ability to "transfer" the discrete Hilbert transform to the Banach space setting (Theorem 5.2). This circle of ideas in conjunction with Corollary 4.4 will enable us to derive an explicit expression for the spectral decomposition of a trigonometrically well-bounded operator $U$ on a Banach space $\mathfrak{X}$ in terms of strong limits of appropriate averages for the powers of $U$ (Theorem 5.3). For these considerations it will be convenient to state beforehand for ready reference the following operator-valued variant of Fejér's Theorem (see [3], Theorem (3.10).(i)).

THEOREM 5.1. Suppose that $U$ is a trigonometrically well-bounded operator on a Banach space $\mathfrak{X}$, and $E(\cdot)$ is the spectral decomposition of $U$. Let $f \in \mathrm{BV}(\mathbb{T})$, and define $f^{\ddagger} \in \mathrm{BV}([0,2 \pi])$ by putting

$$
f^{\ddagger}(t)=\frac{1}{2}\left\{\lim _{s \rightarrow t^{+}} f\left(e^{i s}\right)+\lim _{s \rightarrow t^{-}} f\left(e^{i s}\right)\right\} .
$$

Then the formal series $\sum_{k=-\infty}^{\infty} \widehat{f}(k) U^{k}$ is $(C, 1)$ summable in the strong operator topology to (that is, the sequence $\left\{\sum_{k=-n}^{n}(1-|k| /(n+1)) \widehat{f}(k) U^{k}\right\}_{n=1}^{\infty}$ converges in the strong operator topology to $) \int_{[0,2 \pi]}^{\oplus} f^{\ddagger}(t) d E(t)$.

We now pass to the central result of this section.

TheOREm 5.2. Let $\mathfrak{X}$ be a Banach space, and suppose that $T \in \mathfrak{B}(\mathfrak{X})$ is invertible. Let

$$
\mathcal{W}=\left\{\sum_{0<|k| \leq n}\left(1-\frac{|k|}{n+1}\right) \frac{z^{k} T^{k}}{k}: n \in \mathbb{N}, z \in \mathbb{T}\right\} .
$$

Then the following conditions are equivalent:

(i) $T$ is trigonometrically well-bounded.

(ii) The closure of $\mathcal{W}$ in the strong operator topology of $\mathfrak{B}(\mathfrak{X})$ is compact in the strong operator topology.

(iii) The closure of $\mathcal{W}$ in the weak operator topology of $\mathfrak{B}(\mathfrak{X})$ is compact in the weak operator topology.

Let $\varrho(T)=\sup \|q(T)\|$, where the supremum is extended over all trigonometric polynomials $q$ such that $\|q\|_{\mathrm{BV}(\mathbb{T})} \leq 1$. Then

$$
\varrho(T)<\infty \quad \text { if and only if } \mathfrak{s}(T) \equiv \sup \{\|S\|: S \in \mathcal{W}\}<\infty,
$$


and in this case

$$
\varrho(T) \leq 1+\frac{\mathfrak{s}(T)}{2 \pi}, \quad \mathfrak{s}(T) \leq 8 \pi \varrho(T) .
$$

If $T$ is trigonometrically well-bounded, then for each $z \in \mathbb{T}$, the sequence

$$
\left\{\sum_{0<|k| \leq n}\left(1-\frac{|k|}{n+1}\right) \frac{z^{k} T^{k}}{k}\right\}_{n=1}^{\infty}
$$

converges in the strong operator topology of $\mathfrak{B}(\mathfrak{X})$.

If $\mathfrak{X}$ is reflexive, then in order for $T$ to be trigonometrically well-bounded it is necessary and sufficient that $\mathfrak{s}(T)$ in $(5.2)$ be finite.

Proof. Returning to the function $\phi \in \mathrm{BV}(\mathbb{T})$ defined in (3.6), for each $w \in \mathbb{T}$, let $\phi_{w} \in \mathrm{BV}(\mathbb{T})$ be the function given by $\phi_{w}(z)=\phi(w z)$ for all $z \in \mathbb{T}$. It is clear that, in the notation of (3.8), for $n \in \mathbb{N}, w \in \mathbb{T}$, the trigonometric polynomial $\kappa_{n} * \phi_{w}$ is described by

$$
\left(\kappa_{n} * \phi_{w}\right)(z)=\sigma_{n}(w z) \quad \text { for all } z \in \mathbb{T} \text {. }
$$

Thus,

$$
\left(\kappa_{n} * \phi_{w}\right)(T)=\sum_{0<|k| \leq n}\left(1-\frac{|k|}{n+1}\right) \frac{w^{k} T^{k}}{k}
$$

and so

$$
\mathcal{W}=\left\{\left(\kappa_{n} * \phi_{w}\right)(T): n \in \mathbb{N}, w \in \mathbb{T}\right\} .
$$

Moreover, it is evident from (3.10) and (5.5) that

$$
\sup \left\{\left\|\kappa_{n} * \phi_{w}\right\|_{\mathrm{BV}(\mathbb{T})}: n \in \mathbb{N}, w \in \mathbb{T}\right\} \leq 2 \sup \left\{\left\|\sigma_{n}\right\|_{\mathrm{BV}(\mathbb{T})}: n \in \mathbb{N}\right\} \leq 8 \pi
$$

Using this and (5.7), together with Proposition 2.5, we see that (i) implies (ii).

It is evident that (ii) implies (iii), since the strong operator topology contains the weak operator topology. So to complete the chain of equivalences we now observe that (i) follows from (iii) by Corollary 2.8 and Theorem 2.1.

The assertions in (5.2) and (5.3) are immediate from (2.7) and (5.8).

Turning to the conclusion regarding strong convergence of the sequence in (5.4), we suppose that $T$ is trigonometrically well-bounded, with spectral decomposition $E(\cdot)$. Since $z T$ is also trigonometrically well-bounded for each $z \in \mathbb{T}$, we can assume without loss of generality that $z=1$. We now apply the operator-valued variant of Fejér's Theorem in Theorem 5.1 to $T$ and the function $\phi \in \mathrm{BV}(\mathbb{T})$ defined in (3.6), and thereby infer that, in the strong operator topology,

$$
\left\{\sum_{0<|k| \leq n}\left(1-\frac{|k|}{n+1}\right) \frac{T^{k}}{k}\right\}_{n=1}^{\infty} \rightarrow \int_{[0,2 \pi]}^{\oplus} \phi\left(e^{i t}\right) d E(t) .
$$


For the final conclusion of Theorem 5.2, we need only apply Theorem 2.1 in the case of reflexive $\mathfrak{X}$ to $(5.2)$.

REMARK 5.1. The usual ergodic Hilbert transform associated with an invertible operator $T$ is the formal series $\sum_{0<|k|<\infty} T^{k} / k$, to be summed if possible as the limit in an appropriate topology of the sequence of balanced partial sums (Hilbert averages of $T$ )

$$
\left\{\sum_{0<|k| \leq n} \frac{T^{k}}{k}\right\}_{n=1}^{\infty}
$$

When $T$ is a unitary operator on a Hilbert space, the sequence (5.9) does indeed converge in the strong operator topology via the spectral theorem (see [8], Corollary (11)), and hence will also converge in the strong operator topology if $T$ is a power-bounded invertible operator on a Hilbert space, since such an operator is similar to a unitary. More generally, if $T$ is a powerbounded trigonometrically well-bounded operator on a Banach space, then the sequence in (5.9) will converge in the strong operator topology (see [3], Theorem (3.11), or, for even greater generality, (6.8) and Remark 6.1). In the Banach space setting it is, of course, elementary that if the sequence (5.9) converges in the strong operator topology, then its corresponding sequence of $(C, 1)$-means

$$
\left\{\sum_{0<|k| \leq n}\left(1-\frac{|k|}{n+1}\right) \frac{T^{k}}{k}\right\}_{n=1}^{\infty}
$$

will also converge in the strong operator topology to the same limit.

TheOREM 5.3. Suppose that $U$ is a trigonometrically well-bounded operator on a Banach space $\mathfrak{X}$, and $E(\cdot)$ is the spectral decomposition of $U$. Then for each $\lambda$ such that $0 \leq \lambda<2 \pi$, we have, with all convergence in the strong operator topology of $\mathfrak{B}(\mathfrak{X})$,

$$
\begin{aligned}
E(\lambda)= & (2 \pi i)^{-1}\left\{\lambda i I-S_{\lambda}+S_{0}\right\} \\
& +\lim _{n} \frac{1}{n} \sum_{k=1}^{n}\left(1-\frac{k}{n+1}\right) e^{-i k \lambda} U^{k}+\lim _{n} \frac{1}{n} \sum_{k=1}^{n}\left(1-\frac{k}{n+1}\right) U^{k},
\end{aligned}
$$

where (as justified by Theorem 5.2) we have used the notation

$$
S_{\lambda}=\lim _{n} \sum_{0<|k| \leq n}\left(1-\frac{|k|}{n+1}\right) \frac{e^{-i k \lambda}}{k} U^{k} \quad \text { for } 0 \leq \lambda<2 \pi .
$$

Proof. When $\lambda=0,(5.10)$ reduces to (4.4) for $\lambda=0$. So we assume that $0<\lambda<2 \pi$. Let $f \in \mathrm{BV}(\mathbb{T})$ be the characteristic function of the arc 
$\left\{e^{i s}: 0 \leq s \leq \lambda\right\}$. Then, in the notation of Theorem 5.1,

$$
f^{\ddagger}(t)= \begin{cases}1 / 2 & \text { if } t=0, \lambda, \text { or } 2 \pi, \\ 1 & \text { if } 0<t<\lambda, \\ 0 & \text { if } \lambda<t<2 \pi .\end{cases}
$$

From this, easy direct calculations show that

$$
\int_{[0,2 \pi]}^{\oplus} f^{\ddagger}(t) d E(t)=\frac{1}{2}\left\{E(\lambda)+E\left(\lambda^{-}\right)\right\}-\frac{1}{2} E(0) .
$$

We now apply Theorem 5.1 to $f$. This step, together with the straightforward calculation of the Fourier coefficients of $f$, shows, after taking account of the strong convergence of the sequence in (5.4), that

$$
(2 \pi i)^{-1}\left\{\lambda i I-S_{\lambda}+S_{0}\right\}=\frac{1}{2}\left\{E(\lambda)+E\left(\lambda^{-}\right)\right\}-\frac{1}{2} E(0) .
$$

Adding $E(\lambda) / 2$ to both sides of (5.11), and transposing, we can call upon (4.4) to obtain (5.10).

6. Uniformly bounded ergodic and Hilbert averages. Throughout this section, $\mathfrak{X}$ will be an arbitrary Banach space, and $T \in \mathfrak{B}(\mathfrak{X})$ an invertible linear operator. We shall examine the special circumstances under which the "weighted" averages of $T$, as considered in Sections 4, 5, can be replaced by "unweighted" ergodic averages and Hilbert averages which are uniformly bounded. The variational results for the trigonometric polynomials associated with ergodic averages (in (3.13) and Proposition 3.2), along with the variational result for the trigonometric polynomials associated with Hilbert averages (in (3.9)), show that an analysis purely at the level of scalar-valued functions cannot be useful for describing the context of uniformly bounded, unweighted operator averages which we now wish to consider. For our present purposes, we begin with the following vectorvalued version of Bernstein's Inequality, which follows from the classical statement of Bernstein's Inequality ([7], Proposition B) by applying an arbitrary bounded linear functional in the vector-valued setting.

Proposition 6.1. Suppose that $N \in \mathbb{N},\left\{x_{k}\right\}_{k=-N}^{N} \subseteq \mathfrak{X}$, and $q: \mathbb{T} \rightarrow \mathfrak{X}$ is the $\mathfrak{X}$-valued trigonometric polynomial given by

$$
q(z)=\sum_{k=-N}^{N} z^{k} x_{k}
$$

Then

$$
\sup _{t \in \mathbb{R}}\left\|\frac{d q\left(e^{i t}\right)}{d t}\right\| \leq N \sup _{z \in \mathbb{T}}\|q(z)\|
$$


Theorem 6.2. Suppose that $\mathfrak{X}$ is a Banach space, and $T \in \mathfrak{B}(\mathfrak{X})$ is invertible. Let

$$
\begin{aligned}
& \alpha_{T}=\sup \left\{\left\|\sum_{0<|k| \leq n} \frac{z^{k} T^{k}}{k}\right\|: n \in \mathbb{N}, z \in \mathbb{T}\right\}, \\
& \beta_{T}=\sup \left\{\left\|\frac{1}{n} \sum_{k=1}^{n} z^{k} T^{k}\right\|+\left\|\frac{1}{n} \sum_{k=1}^{n} z^{-k} T^{-k}\right\|: n \in \mathbb{N}, z \in \mathbb{T}\right\} .
\end{aligned}
$$

Then the following assertions are valid:

(i) If $\alpha_{T}<\infty$, then $\beta_{T}<\infty$.

(ii) If $T$ is trigonometrically well-bounded and $\beta_{T}<\infty$, then conversely $\alpha_{T}<\infty$.

Proof. We have, for all $n \in \mathbb{N}, z \in \mathbb{T}$,

$$
\begin{aligned}
\sum_{0<|k| \leq n}\left(1-\frac{|k|}{n+1}\right) & \frac{z^{k} T^{k}}{k} \\
& =\sum_{0<|k| \leq n} \frac{z^{k} T^{k}}{k}-\frac{1}{n+1} \sum_{0<|k| \leq n} \operatorname{sgn}(k) z^{k} T^{k} .
\end{aligned}
$$

We claim that under the hypotheses of either (i) or (ii), the quantity $\mathfrak{s}(T)$ defined in (5.2) is finite. To see this, observe first that as $n$ runs through $\mathbb{N}$, the expression on the left-hand side of (6.2), which is used in calculating $\mathfrak{s}(T)$, runs through the $(C, 1)$ means of the first sum on the right of $(6.2)$, and consequently $\mathfrak{s}(T) \leq \alpha_{T}$. So our claim for the case of (i) is now evident. Under the hypotheses of (ii), the claim follows immediately from the equivalence of Theorem 5.2(i)-(ii) and the Uniform Boundedness Principle.

In order to demonstrate (i), we now suppose that $\alpha_{T}<\infty$. It follows from this hypothesis, together with (6.2) and the claim just established, that

$$
\sup \left\{\left\|\frac{1}{n} \sum_{0<|k| \leq n} \operatorname{sgn}(k) z^{k} T^{k}\right\|: n \in \mathbb{N}, z \in \mathbb{T}\right\}<\infty .
$$

Moreover, for arbitrary $n \in \mathbb{N}$, application of Proposition 6.1 to the $\mathfrak{B}(\mathfrak{X})$ valued trigonometric polynomial $\sum_{0<|k| \leq n} k^{-1} z^{k} T^{k}$ shows that

$$
\sup _{z \in \mathbb{T}}\left\|\sum_{0<|k| \leq n} z^{k} T^{k}\right\| \leq n \alpha_{T}
$$

whence

$$
\sup \left\{\left\|\frac{1}{n} \sum_{0<|k| \leq n} z^{k} T^{k}\right\|: n \in \mathbb{N}, z \in \mathbb{T}\right\} \leq \alpha_{T} .
$$

Upon combining (6.3) and (6.4), we see that $\beta_{T}<\infty$. 
The proof of (ii) follows from (6.2), the hypothesis that $\beta_{T}<\infty$, and the fact that $\mathfrak{s}(T)<\infty$.

Theorem 6.3. Suppose that $\mathfrak{X}$ is a Banach space, and $T \in \mathfrak{B}(\mathfrak{X})$ is a trigonometrically well-bounded operator with spectral decomposition $E(\cdot)$. Then (in the notation of (6.1)),

$$
\alpha_{T}<\infty \text { if and only if } \beta_{T}<\infty \text {. }
$$

If this is the case, then for $0 \leq \lambda<2 \pi$, we have, with all convergence in the strong operator topology of $\mathfrak{B}(\mathfrak{X})$,

$$
\begin{gathered}
\frac{1}{n} \sum_{k=1}^{n} e^{-i k \lambda} T^{k} \rightarrow E(\lambda)-E\left(\lambda^{-}\right) \quad \text { as } n \rightarrow \infty ; \\
\frac{1}{2 n} \sum_{0<|k| \leq n} e^{-i k \lambda} T^{k} \rightarrow E(\lambda)-E\left(\lambda^{-}\right) \quad \text { as } n \rightarrow \infty ; \\
\Phi_{\lambda} \equiv \int_{[0,2 \pi]}^{\oplus} \phi\left(e^{-i \lambda} e^{i t}\right) d E(t)=\lim _{n} \sum_{0<|k| \leq n} \frac{e^{-i k \lambda} T^{k}}{k}, \\
\text { where } \phi \in \mathrm{BV}(\mathbb{T}) \text { is the function defined in }(3.6) ; \\
E(\lambda)=(2 \pi i)^{-1}\left\{\lambda i I-\Phi_{\lambda}+\Phi_{0}\right\}+\lim _{n} \frac{1}{2 n} \sum_{k=1}^{n} e^{-i k \lambda} T^{k}+\lim _{n} \frac{1}{2 n} \sum_{k=1}^{n} T^{k} .
\end{gathered}
$$

Proof. The equivalence in (6.5) is included in Theorem 6.2. For the remainder of the proof we assume that the equivalent conditions of (6.5) hold. For $n \in \mathbb{N}$, put

$$
\mathcal{A}_{n}=\frac{1}{n} \sum_{k=1}^{n} e^{-i k \lambda} T^{k} .
$$

Clearly, if $x \in \mathfrak{X}$ with $T x=e^{i \lambda} x$, then $\mathcal{A}_{n} x=x$ for all $n$. In view of Theorem 4.2 and the hypothesis $\beta_{T}<\infty$, it now suffices for (6.6) to establish that for all $y \in \mathfrak{X},\left\|\mathcal{A}_{n}\left(I-e^{-i \lambda} T\right) y\right\| \rightarrow 0$. For this purpose, notice first for each $n \in \mathbb{N}$,

$$
\mathcal{A}_{n}\left(I-e^{-i \lambda} T\right) y=\frac{1}{n}\left(e^{-i \lambda} T y-e^{-i(n+1) \lambda} T^{n+1} y\right) .
$$

Since $T$ is trigonometrically well-bounded, a vector-valued application of the Riemann-Lebesgue Lemma (see [2], Theorem (2.11)) shows that $\left\|T^{n} y / n\right\|$ $\rightarrow 0$, and so (6.6) now follows from (6.10). By, for instance, Theorem 5.2, it is evident that $T^{-1}$ is trigonometrically well-bounded along with $T$. Since it is likewise clear from (6.1) that $\alpha_{T}=\alpha_{T^{-1}}$ and $\beta_{T}=\beta_{T^{-1}}$, we can now apply the general result in (6.6) to $T^{-1}$ so as to infer with the aid of Theorem 4.2 that $\left\{n^{-1} \sum_{k=1}^{n} e^{i k \lambda} T^{-k}\right\}_{n=1}^{\infty}$ converges in the strong operator topology to a projection which commutes with and has the same range as $E(\lambda)-E\left(\lambda^{-}\right)$ 
(and hence coincides with $E(\lambda)-E\left(\lambda^{-}\right)$). This outcome, together with (6.6), gives (6.7).

For the proof of (6.8), we observe initially that the hypothesis $\alpha_{T}<\infty$ permits us to infer from [2], Corollary (3.9), that $\left\{\sum_{0<|k|<n} k^{-1} e^{-i k \lambda} T^{k}\right\}_{n=1}^{\infty}$ converges in the strong operator topology. We now define $\phi_{\lambda} \in \mathrm{BV}(\mathbb{T})$ by putting $\phi_{\lambda}(z) \equiv \phi\left(e^{-i \lambda} z\right)$. Hence the Fourier transform $\widehat{\phi}_{\lambda}$ of $\phi_{\lambda}$ is specified by $\widehat{\phi}_{\lambda}(k)=e^{-i k \lambda} / k$ for $k \in \mathbb{Z} \backslash\{0\}$, and $\widehat{\phi}_{\lambda}(0)=0$. Applying this in Theorem 5.1, we see that the sequence $\left\{\sum_{0<|k| \leq n} k^{-1} e^{-i k \lambda} T^{k}\right\}_{n=1}^{\infty}$ converges $(C, 1)$ in the strong operator topology to $\int_{[0,2 \pi]}^{\oplus} \phi\left(e^{-i \lambda} e^{i t}\right) d E(t)$. Since we already know that $\left\{\sum_{0<|k| \leq n} k^{-1} e^{-i k \lambda} T^{k}\right\}_{n=1}^{\infty}$ converges in the strong operator topology, the demonstration of (6.8) is now complete.

Since convergence (respectively, $(C, 1)$ convergence) of a sequence implies its $(C, 1)$ convergence (respectively, $(C, 2)$ convergence), the validity of $(6.9)$ is evident upon combining Theorem 5.3 with the convergence in the strong operator topology of the sequences appearing in (6.6) and (6.8).

Remark 6.1. In [5], Section 4, the $A_{p}$ weight condition of Muckenhoupt is used to give various examples of trigonometrically well-bounded operators $T$ on reflexive $L^{p}$-spaces of measures such that $T$ is not power-bounded, but the equivalent conditions of (6.5) are satisfied.

Acknowledgements. The authors are grateful to the referee for comments which have improved the exposition of this paper.

\section{References}

[1] E. Berkson and T. A. Gillespie, AC functions on the circle and spectral families, J. Operator Theory 13 (1985), 33-47.

[2] —, - Fourier series criteria for operator decomposability, Integral Equations Operator Theory 9 (1986), 767-789.

[3] -, 一, Stečkin's theorem, transference, and spectral decompositions, J. Funct. Anal. 70 (1987), 140-170.

[4] -, 一, Spectral decompositions and vector-valued transference, in: Analysis at Urbana II (Proc. Special Year in Modern Analysis at the University of Illinois, 198687), London Math. Soc. Lecture Note Ser. 138, Cambridge Univ. Press, Cambridge, 1989, 22-51.

[5] - - - Mean-boundedness and Littlewood-Paley for separation-preserving operators, Trans. Amer. Math. Soc. 349 (1997), 1169-1189.

[6] E. Berkson, T. A. Gillespie and P. S. Muhly, Abstract spectral decompositions guaranteed by the Hilbert transform, Proc. London Math. Soc. (3) 53 (1986), 489-517.

[7] A. Browder, On Bernstein's Inequality and the norm of hermitian operators, Amer. Math. Monthly 78 (1971), 871-873.

[8] J. T. Campbell, Spectral analysis of the ergodic Hilbert transform, Indiana Univ. Math. J. 35 (1986), 379-390. 
[9] H. R. Dowson, Spectral Theory of Linear Operators, London Math. Soc. Monogr. 12, Academic Press, New York, 1978.

[10] N. Dunford and J. T. Schwartz, Linear Operators. Part I: General Theory, Pure Appl. Math. 7, Interscience, New York, 1958.

[11] R. Goldberg, Methods of Real Analysis, Blaisdell, New York, 1965.

[12] U. Krengel, Ergodic Theorems, de Gruyter Stud. Math. 6, de Gruyter, Berlin, 1985.

[13] A. Zygmund, Trigonometric Series, Vol. 1, 2nd ed., Cambridge Univ. Press, Cambridge, 1959.

Department of Mathematics

University of Illinois

1409 W. Green St.

Urbana, IL 61801, U.S.A.

E-mail: berkson@math.uiuc.edu
Department of Mathematics and Statistics University of Edinburgh James Clerk Maxwell Building Edinburgh EH9 3JZ, Scotland E-mail: t.a.gillespie@edinburgh.ac.uk

Received March 2, 1999

Revised version July 27, 2000 